\title{
THE ANTITUMOR EFFICACY OF CISPLATIN IN COMBINATION WITH TRIPTORELIN AND EXEMESTANE THERAPY FOR AN OVARIAN CANCER ASCITES MODEL IN WISTAR RATS
}

\author{
I.G. Tkalia*, L.I. Vorobyova, A.N. Grabovoy, V.S. Svintsitsky, T.O. Tarasova \\ National Cancer Institute, Kyiv 03022, Ukraine
}

\begin{abstract}
Aim: To study antitumor activity of triptorelin — agonist of gonadotropin-releasing hormone and exemestane — inhibitor of aromatase in combination with cisplatin on the model of receptor-positive for estrogens and progesterone malignant transplantable ascites ovarian tumor (OT); to assess tumor response to treatment and VEGF expression in tumor cells under different combinations of cytostatic and hormonal drugs. Materials and Methods: 36 female Wistar rats, which underwent intraperitoneal transplantation of ascites OT $\left(5 \times 10^{6}\right.$ cells per animal), have been involved in the study. Rats were distributed into 4 groups ( 9 rats in each group): group 1 - animals, which received combination of cisplatin and triptorelin; group 2 - rats treated with combination of cisplatin and exemestane; group 3 animals, which were administered with combination of cisplatin, triptorelin and exemestane; group 4 - rats, which received combination of triptorelin and exemestane. Histological study with assessment of treatment pathomorphosis in OT and immunohistochemical study have been carried out to analyze VEGF expression in OT cells. Survival of animals has been evaluated. Results: Combination of cytostatic agent with triptorelin or exemestane has demonstrated significantly higher rates of treatment pathomorphosis $(\mathbf{1 0 . 1} \pm \mathbf{0 . 1 \%}$ and $16.2 \pm 0.3 \%$, respectively) and antiangiogenic activity in OT (21.4 $\pm 1.4 \%$ and $15.0 \pm 1.3 \%$, respectively), as well as the highest survival of animals (100.0 and $\mathbf{8 5 . 7 \%}$, respectively) as compared with the same in rats treated in regimen of monotherapy with cisplatin, triptorelin, exemestane or by combination of hormonal drugs. Among animals treated by combination of cytostatic drug with triptorelin, two were cured (22.2\%), and among rats, which received cisplatin and exemestane, one animal (11.1\%) was cured. Conclusions: Triptorelin and exemestane increase antitumor activity of cisplatin in respect to the transplantable malignant ascites OT and significantly increase survival of animals, especially when triptorelin and cisplatin are used in combination.
\end{abstract}

Key Words: transplantable ascites ovarian tumor, rat, cisplatin, agonist of gonadotropin-releasing hormone triptorelin, inhibitor of aromatase exemestane, treatment pathomorphosis, VEGF, survival.

At present, hormonal factors take a significant role in the pathogenesis of ovarian cancer (OC). Continuous ovulation resulting from reduction of number of pregnancies and deliveries in women, infertility of different genesis and application of medical drugs stimulating ovulation are referred to those factors. However, hormonal factors represent a part in complex mechanism of endocrine-metabolic and molecular-genetic disorders [1-5]. The results of many studies have shown that $\mathrm{OC}$ has excessive heterogenicity of molecularbiological markers and their complex interaction with factors of microenvironment including the factor of neoangiogenesis (vascular endothelial growth factor VEGF) [6-8]. Treatment effectiveness in OC patients is significantly dependent on the molecular-biologic characteristics of the tumor [9]. The problems of hormone-dependence of $\mathrm{OC}$ and hormonal therapy use for this category of patients are still disputable at present time. In the previous article we demonstrated data of study of animals with transplantable malignant ascites estrogen and progesterone receptor-positive ovarian tumor (OT) treated in regimen of cisplatin, triptorelin and exemestane monotherapy [10]. We have shown that among rats, which were treated in monotherapy

\footnotetext{
Submitted: October 23, 2014

*Correspondence: E-mail: j.tkalya@gmail.com Abbreviations used: ERs - estrogens receptors; Gn-RH - gonadotropin-releasing hormone; ILS - increase of life-span; MLS mean of life-span; OC - ovarian cancer; OT - ovarian tumor; PRs - progesterone receptors; RPVTT - relative part of viable tumor tissue; VEGF - vascular endothelial growth factor.
}

regimen, the strongest antiangiogenic activity in cells of OT has been determined with use of hormonal drugs, at the same time, the highest level of treatment pathomorphosis in OT - histological tumor response - has been observed at treatment with cisplatin [10]. Survival of animals treated with cisplatin was higher, than the same in rats treated with hormonal drugs; however, differences were not significant [10]. In the following article, we will present the results of study of animals with transplantable OT, which were treated with combination of cisplatin and hormonal drugs - triptorelin and exemestane. Also we will compare the results of treatment of animals in monotherapy regimen and with use of combination of these agents.

Aim of study - to investigate antitumor activity of agonist of gonadotropin-releasing hormone $(\mathrm{Gn}-\mathrm{RH})$ - triptorelin and aromatase inhibitor exemestane in combined use with cisplatin on the model of transplantable malignant ascites estrogen and progesterone receptor-positive OT; to evaluate treatment pathomorphosis and VEGF expression in tumor cells using different combinations of cytostatic agent and hormonal drugs; to compare the results in treatment of rats with the use of both monotherapy and combined treatment by mentioned agents.

\section{MATERIALS AND METHODS}

Animals. 36 female Wistar rats (130-150 g b. w.) bred in the vivarium of R.E. Kavetsky Institute of Experimental Pathology, Oncology and Radiobiology, NAS of Ukraine (Kyiv, Ukraine) have been involved in the experiment. All procedures have been carried 
out in accordance with the recommendations for conducting the medical-biological studies with use of laboratory animals.

Model of tumor - strain of ascites malignant OT. Characteristics of tumor and methods of transplantation of OT to the animals were described earlier [10]. Transplantable ascitic liquid was immunocytochemically tested for the determination of expression of steroid hormones receptors in the tumor cells.

Immunocytochemical analysis of the expression of estrogens (ERs) and progesterone receptors (PRs) in the cells of transplantable OT has been carried out in accordance with conventional strategy using monoclonal antibodies specific to the ER - anti-Rat Estrogen Receptor a (Clone 6F11, Thermo Scientific, USA), PR - anti-Rat Progesterone Receptor (Clone PR-AT 4.14, Thermo Scientific, USA). For the total assessment of ERs, PRs expression in OT, method applied by us earlier has been used [10].

Therapy. Cisplatin $(P)$ has been used as solution for injection $(0.5 \mathrm{mg} / \mathrm{ml}$ in $100 \mathrm{ml})$. Triptorelin $(\mathrm{T})$ was used in the form of dry substance of triptorelin acetate $0.1 \mathrm{mg}+$ mannite $10 \mathrm{mg}$ and solvent in the form of sodium chloride $0.9 \% 1 \mathrm{ml}$. Exemestane (E) was applied in the form of pills, 1 pill contains $25 \mathrm{mg}$ of exemestane. Treatment with exemestane was started on the day of transplantation and has been carried out during 10 days, with cisplatin and triptorelin -48 hours after transplantation during 5 days. Treatment drugs were applied to animals in treatment doses according to the current method recommendations and data of previously conducted studies [10].

Rats were divided into 4 groups, 9 rats in each group (Table 1).

Table 1. Groups of animals according to treatment regimen

\begin{tabular}{|c|c|}
\hline Animals, $\mathrm{n}=36$ & Treatment \\
\hline Group 1 & Combination of cisplatin and triptorelin. \\
\hline$(P+T)$ & Cisplatin intraperitoneally $1.0 \mathrm{mg} / \mathrm{kg}-0.15 \mathrm{mg} /$ \\
\hline \multirow[t]{2}{*}{$n=9$} & $\begin{array}{l}\text { rat. day }{ }^{-1} \text { or } 0.3 \mathrm{ml}+0.3 \mathrm{ml} \text { of physiological solution/ } \\
\text { rat. day }{ }^{-1} \text { during } 5 \text { days. }\end{array}$ \\
\hline & $\mathrm{g} / \mathrm{rat} \cdot \mathrm{day}-$ \\
\hline $\begin{array}{l}\text { Group } 2 \\
(\mathrm{P}+\mathrm{E})\end{array}$ & doses mentioned above \\
\hline $\mathrm{n}=9$ & $0 \mathrm{mkg} / \mathrm{kg})+0.5 \mathrm{ml}$ physi- \\
\hline & \\
\hline Group 3 & Combination of cisplatin (during 5 days), triptorelin (for \\
\hline$(P+T+E)$ & 5 days) and exemestane (during 10 days) in the doses \\
\hline & \\
\hline $\begin{array}{l}\text { Group } 4 \\
(T+E), n=9\end{array}$ & $\begin{array}{l}\text { Combination of triptorelin (during } 5 \text { days) and exemes- } \\
\text { tane (for } 5 \text { days) in the doses mentioned above }\end{array}$ \\
\hline
\end{tabular}

Observation has been started in 24 hours after the last injection of exemestane. Died or euthanized animals underwent autopsy for macroscopic evaluation of tumor process spread and pathological changes of internal organs. Cancer-changed organs of peritoneal cavity and pelvis of rats were removed for histological and immunohistochemical studies. In order to evaluate dynamics of tumor process objectively, studied animals were euthanized by one rat from each group through decapitation on $14^{\text {th }}, 17^{\text {th }}$ and $19^{\text {th }}$ day of observation. Moreover, macroscopic study of internal organs in intact rats was carried out for the objective analysis of pathological changes in organs of peritoneal cavity and pelvis of the studied animals. Antitumor effect was evaluated as presented earlier [10].

Toxic effect of antitumor drugs has been assessed by degrowth of spleen (indirect signs of total and hematological toxicity), which was weighed on torsion scales, and by macroscopic study of pathological changes in liver and kidneys of animals.

Histological analysis. Obtained material was fixed in buffered $10 \%$ formalin with $\mathrm{pH} 7.4$ and condensed in paraffin using histoprocessor Histos-5 (Milestone, Italy). From paraffin blocks, 5 micron thickness histological sections were prepared with the help of microtome Microm HM325 (Thermo Scientific, Germany). Sections were stained with hematoxylin-eosin (H \& E) for general assessment of tumor. Analysis of histological response - treatment pathomorphosis in tumor tissues was carried out according to K.A. Galakhin method [11] by determining a relative part of viable tumor tissue (RPVTT) in percentage.

Immunohistochemical study of VEGF expression in the OT cells has been carried out using of monoclonal rat antibody specific to the VEGF - anti-VEGF Ab-1 RB-222 Polyclonal (Thermo Scientific, USA). For evaluation of VEGF expression were assessed by semiquantitative method via calculation of the number of positively stained cells in percentage [10].

Obtained results were studied and photographed using microscope Nikon Eclipse 80i with camera DS$5 S M c / L 2$ and optical magnification $\times 400$.

Statistical analysis. Processing of the results of study has been carried out using program package Statistica 6.0. Parametric statistics including method of Student's $t$-criterion was used. Survival of animals has been analyzed by Kaplan-Meier, log-rank criterion was used for paired comparisons, criterion $\chi^{2}-$ for multiple comparisons between groups. Data were considered statistically significant at $p<0.05$.

\section{RESULTS}

In accordance with immunocytochemical study the grade of ERs and PRs expression in OT cells was high $-60 \%$ each.

Animals, which received combination of cisplatin and triptorelin (Group 1, P + T), and were euthanized on days 14, 17 and 19, had no ascites. No tumor lesions in abdominal cavity and pelvis were detected macroscopically. Omentum was lysed, single papillary inclusions on serous tissues of intestine and in ovaries were observed in one of 3 rats. Other 4 rats, which died in later terms of observation (Table 2), had serous or hemorrhagic ascites. The volume of ascitic liquid has constituted $10.1 \pm 5.3 \mathrm{ml}$ and was lower as compared with the same in animals, which received cisplatin, triptorelin or exemestane monotherapy [10]. Spread of tumor process and level of involvement of organs of abdominal cavity and pelvis were lower as compared with the same ones using cisplatin and hormonal drug monotherapy [10], distinctive feature - small size of ovaries with insignificant papillary inclusions, ovaries of two rats had no visual signs of tumor. Liver and 
kidneys had no pathological changes. In one rat, which died after 60 days, liver decreased in size, had an ochreyellow tint, and was of flaccid consistency. Mean mass of spleen decreased on $28.5 \%$ ( $800.4 \pm 7.2 \mathrm{mg}$ ) that was significantly lower as compared with weight of spleen in animals of the control group $(p<0.05)$ [10]. At the same time, this rate did not differ significantly from the same one in rats, which received cisplatin monotherapy [10]. Histological analysis has shown large areas of necrosis and hemorrhages, accumulation of fibrous masses in tumor lesion (Figure). Cellular compound of OT was characterized by significant polymorphism, with larger part of degenerating cells and cells with pyknotic nuclei. Only single cells with equally stained cytoplasm and large round nuclei have been found. Mean RPVTT and VEGF expression in lesions of adenocarcinoma overgrowths were significantly low (10.1 \pm 0.1 and $21.4 \pm 1.4 \%$, respectively) as compared with the same ones in OT of rats in control group and animals treated by monotherapy with cisplatin, triptorelin and exemestane $(p<0.01, p<0.01, p<0.01, p<0.01$, respectively) [10] (Table 3). Besides, significantly high survival of animals of this group has been observed as compared with the same one in rats, which were administered monotherapy using cisplatin, triptorelin and exemestane and the animals in the control group $(p<0.01, p=0.01, p=0.01, p<0.01$, respectively) [10]. At the same time, increase of life-span (ILS) has constituted $213.8 \%$. Two animals (22.2\%) have been treated and are still alive ( 545 days) without any signs of disease (see Table 2).

Table 2. Survival rates of rats with transplantable OT

\begin{tabular}{|c|c|c|c|c|c|c|c|}
\hline \multirow{2}{*}{$\begin{array}{l}\text { Groups } \\
\text { of animals }\end{array}$} & \multirow{2}{*}{$\begin{array}{l}\text { ILS, } \\
\%\end{array}$} & \multirow[t]{2}{*}{ MLS, days } & \multirow{2}{*}{$\begin{array}{l}\text { Min } \\
\text { LL, } \\
\text { days }\end{array}$} & \multirow{2}{*}{$\begin{array}{l}\text { Max LL, } \\
\text { days }\end{array}$} & \multirow[t]{2}{*}{$\begin{array}{c}\text { OS, } \\
\%\end{array}$} & \multicolumn{2}{|c|}{$\begin{array}{c}\text { Cured } \\
\text { animals }\end{array}$} \\
\hline & & & & & & $n$ & $\%$ \\
\hline $\begin{array}{l}\text { Group 1 } \\
(\mathrm{P}+\mathrm{T}) \\
\mathrm{n}=9(100 \%)\end{array}$ & $213.9^{*}$ & $\begin{array}{c}43.0(147.2)^{\star \star \star} \\
\pm 11.3\end{array}$ & 16 & $88(545)^{\star \star \star}$ & 100.0 & 2 & 22.2 \\
\hline $\begin{array}{l}\text { Group } 2 \\
(P+E) \\
n=9(100 \%) \\
\text { Group 3 }\end{array}$ & $205.8^{\star \star}$ & $\begin{array}{c}41.9(75.8)^{\star \star \star} \\
\pm 10.0\end{array}$ & 16 & $86(365)^{\star \star \star}$ & $85.7 \pm 13.2$ & 1 & 11.1 \\
\hline $\begin{array}{l}(P+T+E) \\
n=9(100 \%) \\
\text { Group } 4\end{array}$ & 40.1 & $19.2 \pm 1.3$ & 16 & 27 & $44.3 \pm 15.4$ & 0 & 0 \\
\hline $\begin{array}{l}(T+E) \\
n=9(100 \%)\end{array}$ & 31.4 & $18.0 \pm 1.7$ & 15 & 26 & $32.2 \pm 14.0$ & 0 & 0 \\
\hline
\end{tabular}

Note: ILS - increase of life-span; Max LL - maximal length of life; Min LL minimal length of life; MLS - mean of life-span; OS - overall survival; *living rats with life-span more than 545 days have not been taken into account; **euthanized rat with 365 day life-span has not been taken into account; ${ }^{* * *}$ calculations with living rats of group 1 and one euthanized rat of group 2 are indicated in brackets.

Animals, which received combination of cisplatin and exemestane (Group 2, P + E) and were euthanized on days $14,17,19$, had no ascites, the same as in rats of group 1. Macroscopic assessment has detected insignificant tumor lesions in omentum and uterine appendages. At the same time, ovaries were small in size as compared with the same ones in animals of the control group and in rats treated by cisplatin [10]. Rats, which have died in later terms of observation, had hemorrhagic ascites. The volume of ascitic liquid has constituted $8.0 \pm 1.2 \mathrm{ml}$.
Among rats which died, two rats had no visual signs of tumor involvement in abdominal cavity organs and pelvis. In other three animals, which have died on days 53; 85 and 86 of observation, spread and level of involvement of internals in tumor process were significantly lower as compared with the same ones in rats, which received cisplatin monotherapy [10] and identical as compared with the animals which were administered a combination of cisplatin and triptorelin. However, ovaries in these died rats had form of solid tumor as compared to everting papillary tumors, which were observed in animals of group 1 , and also in rats treated by monotherapy and in the animals of the control group [10]. During visual assessment of liver and kidneys in all rats of this group, flaccid consistency and slight ochre-yellow coloration of the liver were observed in 2 animals and their kidneys had yellow specks on incision. Decrease of spleen weight has constituted $29.3 \%$ that had no significant differences as compared with the same one in rats, which received cisplatin monotherapy [10]. Histological study of adenocarcinoma overgrowths has determined widespread necrosis, as well as significant inflammatory infiltration, hemorrhages, multiple lesions of angiomatosis and fibrosis (see Figure). Among necrosis lesions, fibrous layers have been determined, sometimes with hyalinosis and myxomatosis. Mean RPVTT has constituted $16.2 \pm 0.3 \%$, VEGF expression was low (15.0 $\pm 1.3 \%$ ) (see Table 3). Statistical analysis has determined significant differences between these indices as compared with the same ones in OT of rats of group 1, in animals treated by monotherapy and in rats of the control group $(p<0.01)$ [10]. When analyzing the results in Table 2, we have determined significantly high survival of animals of this group as compared with the same one in rats of the control group and animals which received monotherapy using triptorelin and exemestane $(p=0.02, p=0.04, p=0.03$, respectively) [10]. However, survival rates of animals in group 2 were higher as compared with the same ones in rats treated with cisplatin [10], and lower as compared with animals of group 1. At the same time, statistical significance of such differences has not been determined $(p=0.08$ and $p=0.4$, respectively). ILS of animals of this group has constituted $205.8 \%$. One rat (11.1\%) has been treated, but on the day 365, a tumor in the right breast was detected in this rat. Autopsy of euthanized animal has not determined macroscopically and histologically any pathological changes considering organs of chest, abdominal cavity, pelvis and retroperitoneal space. Tumor in the right breast, $4.0 \times 3.5 \mathrm{~cm}$ in size, has been found - histologically fibroadenoma.

Table 3. Antitumor effectiveness and toxic effect of treatment drugs in different combinations by RPVTT, VEGF expression in cells of OT and change of spleen weight in rats with transplantable OT

\begin{tabular}{lccc}
\hline \multicolumn{1}{c}{ Groups of animals } & RPVTT, \% & VEGF, \% & $\begin{array}{c}\text { Spleen weight } \\
\text { decrease, \% }\end{array}$ \\
\hline Group (C), $\mathrm{n}=9$ & $50.1 \pm 1.6$ & $77.8 \pm 2.8$ & - \\
Group 1 $(\mathrm{P}+\mathrm{T}), \mathrm{n}=7$ & $10.1 \pm 0.1^{\star}$ & $21.4 \pm 1.4^{\star}$ & $28.5^{\star}$ \\
Group 2 (P+E), $\mathrm{n}=8$ & $16.2 \pm 0.3^{\star}$ & $15.0 \pm 1.3^{\star}$ & $29.3^{\star}$ \\
Group 3 (P+T+E), $\mathrm{n}=9$ & $10.1 \pm 0.3^{\star}$ & $9.4 \pm 1.8^{\star}$ & $38.7^{\star}$ \\
Group 4 (T+E), $\mathrm{n}=9$ & $22.1 \pm 0.4^{\star}$ & $12.2 \pm 0.9^{\star}$ & 13.6 \\
\hline
\end{tabular}

Note: Group (C) - rats of control group; ${ }^{\star} p<0.05$ in comparison with the control group [10]. 

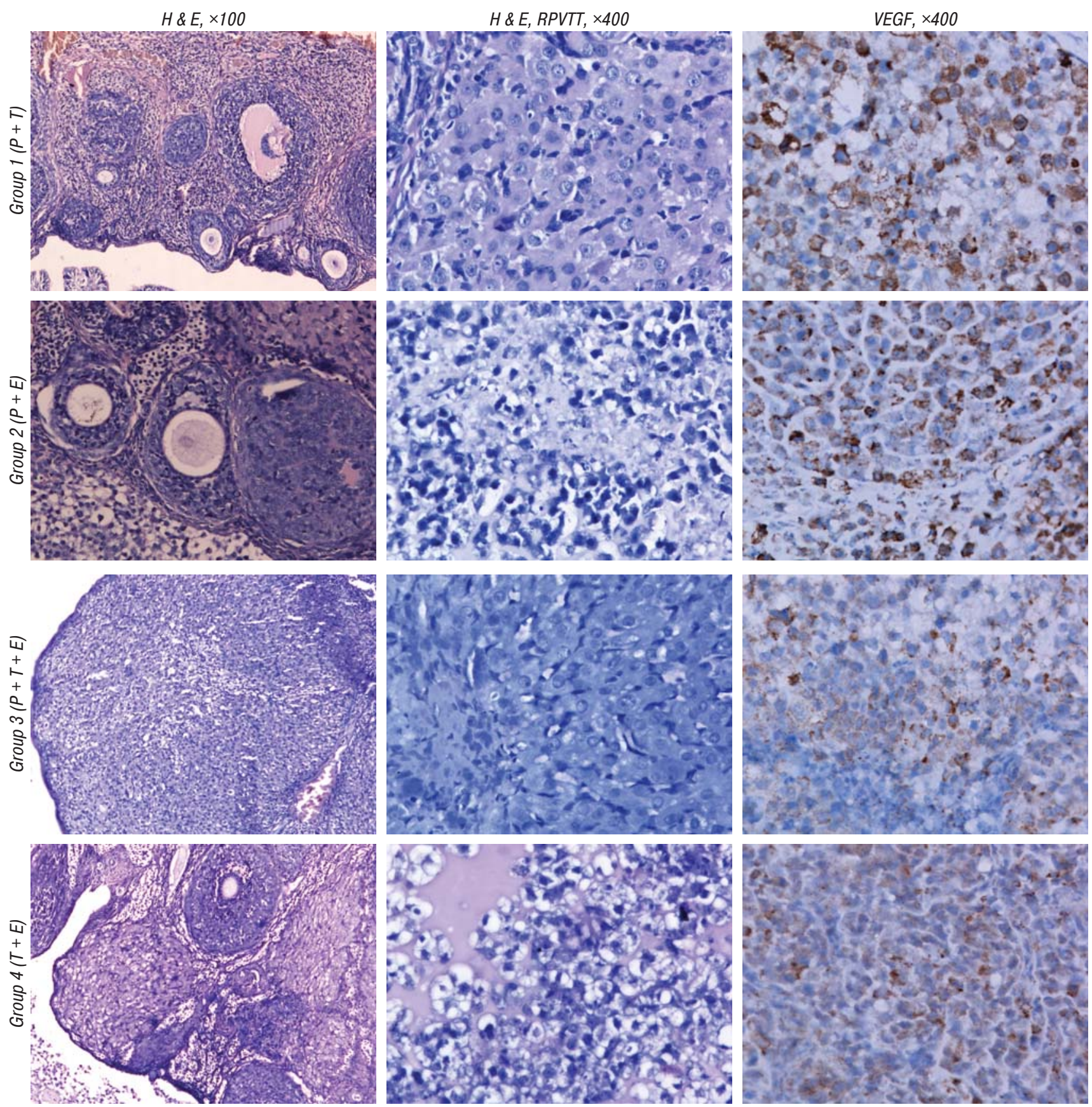

Figure. Morphological and immunohistochemical picture in ovaries of rats with transplantable OT of groups 1-4. The highest rates of treatment pathomorphosis (RPVTT) in OT were noticed in the rats of group 1 (combination of cisplatin and triptorelin) and group 3 (combination of cisplatin + triptorelin + exemestane) $(p<0.05)$, whereas the highest expression of VEGF in cells of OT has been observed in the animals of groups, where the combination of triptorelin and exemestane was used $-3(p<0.05)$ and 4 (significant differences in the level of VEGF expression in OT of this group as compared with rats of group 1, and lack of significance of such differences - with rats of groups 2-3)

Rats treated with combination of cisplatin + triptorelin + exemestane (Group 3, P + T + E) and euthanized on days 14, 17 and 19, also had no ascites, the same as animals of groups 1 and 2. Macroscopic assessment of abdominal cavity organs and pelvis has not shown tumor lesions, but ovaries of two rats had single tumor papillary inclusions with hemorrhages. Animals, which have died in later terms of observation, had hemorrhagic ascites. The volume of ascitic liquid has constituted $6.5 \pm 1.5 \mathrm{ml}$ and was lower as compared with the same one in rats of groups 1-2, in animals of the control group and rats treated using cisplatin, triptorelin and exemestane monotherapy [10]. The fact was interesting that in all died animals, no tumor changes of abdominal cavity organs and pelvis were found, ovaries were atrophic. However, liver enlargement, its flaccid consistency and ochre-yellow coloration have been detected in all rats of this group. Kidneys were also enlarged, flaccid, of grey color with yellow specks on incision and their cortical layer was dilated with signs of swelling. Decrease of spleen weight was the highest $(38.7 \%)$ as compared with the same rate in rats of groups 1-2 and in animals which received cisplatin in monotherapy [10], but differences between them had no significance $(p>0.05)$ (see Table 3$)$. Histological analysis has shown significant degenerative-necrotic changes in OT, which were similar to those in animals of group 1 and rats treated by cisplatin [10] (see Fi- 
gure). RPVTT has constituted $10.1 \pm 0.3 \%$. This rate was the same as one in rats, which received combination of cisplatin and triptorelin, however, when compared with RPVTT of animals, which were treated in regimen of cisplatin monotherapy (11.7 $\pm 0.2 \%$ ) [10], statistical analysis has demonstrated significant differences between mentioned rates $(p<0.01)$ (see Table 3 ). Expression of VEGF in tumor cells was the lowest (9.4 \pm $1.8 \%$ ) and was significantly different as compared with the same in OT in rats of other groups $(p<0.01)$. However, survival rates among rats of this group turned out to be significantly lower, than in animals of groups 1 and $2(p<0.01)$, and had no significant differences as compared with the same rates in rats which were administered cisplatin, triptorelin and exemestane monotherapy [10] ( $p>0.05$ ) (see Table 2). At the same time, these rates were significantly higher as compared with survival rates in animals of the control group [10] $(p=0.01)$. ILS of animals of this group has constituted $40.1 \%$. Obtained results of the study allow assuming that in rats treated with combination of cisplatin + triptorelin + exemestane toxic dystrophy of liver and kidneys has been present with further development of acute hepatocellular and kidney failure, which was likely a direct cause of animals' death.

In rats, which received combination of triptorelin and exemestane (Group 4, T $+E$ ), volume of ascitic liquid has constituted $15.2 \pm 3.4 \mathrm{ml}$, and had no significant differences as compared with the same one in animals treated with triptorelin or exemestane monotherapy [10]. Ascitic liquid was hemorrhagic and serous"lactic". Macroscopic study of rats, which have been euthanized on days 14,17 , and of one rat, which died on day 15 , demonstrated no tumor changes of the abdominal wall, organs of abdominal cavity and pelvis. Atrophic changes in ovaries were determined and one of them had insignificant papillary tumor overgrowths. In other animals, spreading of tumor process in organs of abdominal cavity and pelvis has been observed and its extent correlated directly with terms of observation. At study of liver and kidneys, no pathological changes were determined. Decrease of spleen weight has constituted $13.6 \%$ that had no significant differences in respect to the same rate in rats of control group and animals, which received triptorelin or exemestane monotherapy [10]. Histological study has shown that spreading of tumor in organs, walls of abdominal cavity and pelvis, as well as sizes of tumor tissue in rats of this group were almost twice less, than in control animals, but larger, than in rats of those groups with cisplatin application [10]. In overgrowths of adenocarcinoma, large necrosis and hemorrhages were also observed. Multiple lesions with angiomatosis and strong fibrosis, which was found in uterus, tubes and mesentery of intestine, have been determined in ovaries. Viable cells of adenocarcinoma were quite large, had basophilic cytoplasm and large nuclei, some cells with lucid cytoplasm and vacuoles were determined among them, sometimes with strong pyknotic changes of nuclei. Mean RPVTT has constituted $22.5 \pm 0.4 \%$, this rate was significantly lower, than the same one in control animals and rats, which were treated in regimen of triptorelin or exemestane monotherapy [10] $(p<0.01)$, and significantly higher, than this rate in animals of those groups, where cisplatin was applied $(p<0.01)$. VEGF expression was low (12.2 $\pm 0.9 \%$ ) in small areas of tumor cells, which were arranged by loci, but expression of this marker was not detected in the major part of cells (see Table 3). Statistical analysis has determined significant differences in the level of VEGF expression in OT of this group as compared with rats of group 1, animals of control group and rats which received monotherapy using cisplatin, triptorelin and exemestane [10], and lack of significance of such differences - with rats of groups 2-3. However, survival rates of animals of this group were not high $(32.2 \pm 14.0 \%)$, they were the same as rates in rats treated with triptorelin or exemestane monotherapy [10]. Moreover, there were no significant differences regarding the same rates in control animals [10] $(p=0.2)$. ILS has constituted $31.4 \%$ (see Table 2).

\section{DISCUSSION}

In the previous article [10], we have analyzed the correlation between level of treatment pathomorphosis (RPVTT), expression of VEGF in cells of OT and life-span of animals. Inverse correlation between survival of animals and rate of RPVTT in OT ( $r=-0.3$, $p<0.01$ ), as well as direct correlation between RPVTT and expression of VEGF in tumor cells $(r=0.7, p<0.01)$ have been determined [10]. At the same time, no correlation has been found between survival of animals and VEGF expression in OT $(r=-0.2, p=0.11)$ [10]. Data of correlation analysis have proved such variable histological, immunohistochemical rates and the results of survival of animals treated with different treatment drugs and their combinations.

Thus, as it has been stated before [10], represented model of transplantable OT, with positive hormonal receptor status and VEGF hyperexpression, had aggressive clinical course that has been confirmed by histological and immunohistochemical tests, as well as survival rates of animals. Obtained data have demonstrated effectiveness of cytostatic and hormonal drugs in monotherapy [10] and in different combinations. We have shown that not only cytostatic agent - cisplatin, but also hormonal drugs - triptorelin and exemestane in monotherapy have caused histological response - treatment pathomorphosis in OT that allows us to judge indirectly about antiproliferative and apoptotic activity of these agents [12-15]. Among the animals treated using monotherapy with cisplatin, triptorelin or exemestane, the most pronounced antiangiogenic activity in OT has been observed after administration of hormonal drugs, however, the highest treatment pathomorphosis (RPVTT) in OT was in treatment with cisplatin [10].

The most pronounced antiangiogenic activity in OT cells has been determined in animals treated with combination of hormonal drugs triptorelin and exemestane. At the same time, level of treatment pathomorphosis of tumor and rates of survival of these rats had 
no significant differences as compared with the same ones in animals treated with triptorelin or exemestane monotherapy [10]. For this reason, combination of these hormonal drugs has not shown advantages of their application in monotherapy regimen.

Combination of cytostatic agent with triptorelin or exemestane has shown significantly high rates of treatment pathomorphosis and antiangiogenic activity in OT, as well as the highest survival of animals as compared with the same one in rats treated in regimen of cisplatin, triptorelin, exemestane monotherapy [10] or combination of hormonal drugs.

We have noticed that hormonal drugs had no reliably significant toxic effect as compared with cytostatic agent [10], and combination of cisplatin and triptorelin, cisplatin and exemestane did not exceed significantly toxic impact of cisplatin alone.

In animals, which received combination of three drugs, significantly highest antiangiogenic activity and high grade of treatment pathomorphosis have been observed. It was similar to the same one in rats treated with cisplatin and triptorelin, but animals have died from toxic hepatorenal failure. These data are the evidence of necessity of the further studies for mechanisms of potentiating mutual toxic effect of combination of cisplatin, triptorelin and exemestane depending on tolerable and treatment doses.

The most important result of the study consists in the fact that among animals, which received a combination of cytostatic agent with triptorelin or exemestane, three of them were treated $(P+T-22.2 \%, P+E-11.1 \%)$.

Thus, triptorelin and exemestane increase antitumor activity of cisplatin in the respect of transplantable malignant ascites OT and significantly increase survival of animals, especially when used in combination of cisplatin and triptorelin.

This is our first experimental study in vivo with application of hormonal drugs in monotherapy and in combination with the cytostatic agent on the model of transplantable malignant ascites estrogen and progesterone receptor-positive OT. The results obtained demand necessity and perspective to continue experimental studies in vitro and in vivo with the aim to investigate the mechanisms of hormonal-receptor signals realization in the cells of OC under the effect of different sex hormones and to study the mechanisms of increasing antitumor effect of cisplatin with triptorelin and exemestane. This will probably discover new opportunities for clinical approbation of hormonal therapy use, particularly in the combinations above, in the primary complex treatment of $\mathrm{OC}$ patients.

\section{CONCLUSIONS}

Combination of cytostatic drug with triptorelin or exemestane has demonstrated significantly high rates of treatment pathomorphosis and antiangiogenic activity in OT, as well as the highest survival in animals as compared with one in rats treated in regimen of monotherapy with cisplatin, triptorelin, exemestane or by combination of hormonal drugs.

Triptorelin and exemestane increase antitumor activity of cisplatin in regard to transplantable malignant ascites OT and significantly increases survival of animals, especially when used in combination of cisplatin and triptorelin.

\section{REFERENCES}

1. Disaia PJ, Creasman WT. Clinical gynecologic oncology (3) (transl from Eng, ed. by EG Novikova). M: Rid Elsiver, 2012: 346 p. (in Russian).

2. Landen JCN, Birrer MJ, Sood AK. Early stages of the pathogenesis of ovarian cancer. J Clin Oncol 2008; 26: 149-60.

3. Whittemore AS, Balise RR, Pharoah PD, et al. Oral contraceptive use and ovarian cancer risk among carriers of BRCA1 or BRCA2 mutations. Br J Cancer 2004; 91: 1911-5.

4. Urmancheyeva AF, Kutusheva GF, Ulrich EA. Ovarian tumors (clinics, diagnostics and treatment). St-Petersburg: N-L, 2012. 68 p. (in Russian).

5. Gorbunova VA. Diagnostics and treatment of ovarian cancer. M.: MIA, 2011. 248 p. (in Russian).

6. Osinsky SP, Gluzman DF, Kleeff J, et al. Molecular diagnosis of tumors: fundamentals and practical applications. Monography. K: DIA, 2007. 248 p. (in Ukrainian).

7. Ferrara N. The role of VEGF in the regulation of physiological and pathological angiogenesis. EXS 2005; 94: 209-31.

8. Tkalia IG, Vorobyova LI, Svintsitsky VS, et al. Features of hormonal receptor status, expression of VEGF, HER-2/neu in serous cancer and functional cysts of ovaries. Hormonal homeostasis in patients with recurrent serous ovarian cancer. Klin Onkol 2014; 4: 81-9 (in Ukrainian).

9. Chekhun VF, Lukyanova NYu, Yurchenko OV, et al. The role of expression of the components of proteome in the formation of molecular profile of human ovarian carcinoma A2780 cells sensitive and resistant to cisplatin. Exp Oncol 2005; 27: 191-5 (in Ukrainian).

10. Tkalia IG, Vorobyova LI, Grabovoy AN, et al. Increase of antitumor activity of cisplatin using agonist of gonadotropinreleasing hormone and inhibitor of aromatase on the model of ascites ovarian tumor. Exp Oncol 2014; 36: 184-90.

11. Galakhin KA, Kuryk EG. Treatment pathomorphosis of malignant gastrointestinal tract tumors. K: Naukova Dumka, 2000. 178 p. (in Ukrainian).

12. Kim KY, Choi KC, Auersperg N, et al. Mechanism of gonadotropin-releasing hormone (GnRH)-I and -IIinduced cell growth inhibition in ovarian cancer cells: role of the GnRH-I receptor and protein kinase C pathway. Endocrine-Related Cancer 2006; 13: 211-20.

13. So WK, Cheng JC, Poon SL, et al. Gonadotropinreleasing hormone and ovarian cancer: a functional and mechanistic overview. FEBS J 2008; 275: 5496-511.

14. Losordo DW, Isner JM. Estrogen and angiogenesis: a Review. Arterioscler Thromb Vasc Biol 2001; 21: 6-12.

15. Walker G, MacLeod K, Williams AR, et al. Estrogenregulated gene expression predicts response to endocrine therapy in patients with ovarian cancer. Gynecol Oncol 2007; 106: 461-8. 